subarc $A A_{0}$ of $A B$, and $D$ is $B$ or (if $A_{0} \neq B$ ) any point of the subarc $A_{0} B$ of $A B$, then the subarc $C D$ of $A B$ contains at least one point of $[S]$.

This theorem is tacitly assumed by Lennes in his proof of Theorem 7 (\$4).

All the above mentioned theorems thus hold if the term simple continuous arc is defined without the use of the word "bounded." Using this definition of an arc, I now prove the

Theorem. A simple continuous arc is bounded.

Proof. Suppose $A B$ is an arc which is not bounded. Let [ $\left.S_{1}\right]$ consist of $A$ and all points $S_{1}$ of $A B$ such that the arc $A S_{1}$ is bounded. Let $\left[S_{2}\right]$ consist of all other points of $A B$. By hypothesis both $\left[S_{1}\right]$ and $\left[S_{2}\right]$ exist. No point of $S_{2}$ is between $A$ and a point of $\left[S_{1}\right]$. Since $A B$ is connected, $\left[S_{1}\right]$ contains a limit point $P_{1}$ of $\left[S_{2}\right]$ or $\left[S_{2}\right]$ contains a limit point $P_{2}$ of $\left[S_{1}\right]$. In the first case any triangle $t_{1}$ containing $P_{1}$ contains an arc $a_{1}$ of $A B$ containing $P_{1}$. The arc $a_{1}$ contains a point $Q_{2}$ of $\left[S_{2}\right]$. The $\operatorname{arc} A P_{1}$ of $A B$ is contained in a polygon $p_{1}$. Therefore the subarc $A Q_{2}=A P_{1}+P_{1} Q_{2}$ lies entirely within a polygon (Lennes, Theorem $15, \S 2$ ), and is bounded, contrary to hypothesis. In the second case any triangle $t_{2}$ about $P_{2}$ contains an arc $a_{2}$ containing $P_{2}, a_{2}$ contains a point $Q_{1}$ of $\left[S_{1}\right], A Q_{1}$ is contained in a polygon, and therefore $A P_{2}=A Q_{1}$ $+Q_{1} P_{2}$ is contained in a polygon and is bounded, contrary to hypothesis. Thus in either case the supposition that $A B$ is not bounded leads to a contradiction.

University of Pennsylvania.

\title{
THE TRANSFORMATION OF A REGULAR GROUP INTO ITS CONJOINT.
}

\author{
BY DR. J. E. MCATEE,
}

(Read before the American Mathematical Society October 26, 1918.)

1. Consider a regular substitution group $G$ of order $g$. All the substitutions on the same letters that are commutative with every substitution of $G$ form a group $G^{\prime}$, of order $g$, called the conjoint of $G$. These groups are conjugate.* If $G$ is abelian, $G=G^{\prime}$. In the contrary case the statement that a

* Finite Groups, Miller, Blichfeldt and Dickson, 1916, p. 35. 
substitution $t$ of order 2 exists that transforms $G$ into $G^{\prime}$ occurs in the literature.* Our purpose is to exhibit such a $t$.

Let the substitutions of $G$ be arranged as follows:

$$
G=1, s_{2}, s_{3}, \cdots, s_{e}, s_{k}, \cdots, s_{g},
$$

where $1, s_{2}, s_{3}, \cdots, s_{e}$ is the cyclic group generated by $s_{2}$ $\left(s_{i}=s_{2}{ }^{i-1}, i=2, \cdots, e\right)$. Consider the two square arrays

and

$$
\begin{aligned}
& 1, s_{2}, \quad s_{3}, \quad \cdots, s_{e}, \quad s_{k}, \quad \cdots, s_{g} \\
& s_{2}, s_{2}{ }^{2}, s_{3} s_{2}, \cdots, s_{e} s_{2}, s_{k} s_{2}, \cdots, s_{g} s_{2} \\
& s_{3}, s_{2} s_{3}, s_{3}^{2}, \cdots, s_{e} s_{3}, s_{k} s_{3}, \cdots, s_{g} s_{3} \\
& s_{e}, s_{2} s_{e}, s_{3} s_{e}, \cdots, s_{e}{ }^{2}, s_{k} s_{e}, \cdots, s_{g} s_{e} \\
& s_{k}, s_{2} s_{k}, s_{3} s_{k}, \cdots, s_{e} s_{k}, s_{k}^{2}, \cdots, s_{g} s_{k} \\
& s_{g}, s_{2} s_{g}, s_{3} s_{g}, \cdots, s_{e} s_{g}, s_{k} s_{g}, \cdots, s_{g}{ }^{2}
\end{aligned}
$$

$$
\begin{aligned}
& 1, s_{2}, \quad s_{3}, \quad \cdots, s_{e}, \quad s_{k}, \quad \cdots, s_{g} \\
& s_{2}, s_{2}{ }^{2}, s_{2} s_{3}, \cdots, s_{2} s_{e}, s_{2} s_{k}, \cdots, s_{2} s_{g} \\
& s_{3}, s_{3} s_{2}, s_{3}{ }^{2}, \cdots, s_{3} s_{e}, s_{3} s_{k}, \cdots, s_{3} s_{g} \\
& s_{e}, s_{e} s_{2}, s_{e} s_{3}, \cdots, s_{e}{ }^{2}, s_{e} s_{k}, \cdots, s_{e} s_{g} \\
& s_{k}, s_{k} s_{2}, s_{k} s_{3}, \cdots, s_{k} s_{e}, s_{k}^{2}, \cdots, s_{k} s_{g} \\
& s_{g}, s_{g} s_{2}, s_{g} s_{3}, \cdots, s_{g} s_{e}, s_{g} s_{k}, \cdots, s_{g}{ }^{2}
\end{aligned}
$$

Each row of these squares is a permutation of the elements of the first row. Each of these permutations represents a substitution and we may assume, without loss of generality, that the substitutions thus obtained from the first square are those of $G$. For simplicity in what follows we shall call these substitutions $1, u_{2}, u_{3}, \cdots, u_{g}$. The substitutions $1, u_{2}{ }^{\prime}, u_{3}{ }^{\prime}$, $\cdots, u_{g}{ }^{\prime}$ represented by the second square are those of $G^{\prime} . \dagger$

The substitution represented by the row $G s_{2}$ in the first

* Ibid., p. 46.

† Loc. cit. 
square is

$$
u_{2}=s_{1} s_{2} s_{3} \cdots s_{e} \cdot s_{k} s_{h} s_{v} \cdots s_{i} s_{m} s_{n} \cdots s_{x} s_{q} \cdots,
$$

where $s_{1}=1$. The substitution represented by the row $s_{2} G$ in the second square is

$$
u_{2}^{\prime}=s_{1} s_{2} s_{3} \cdots s_{e} \cdot s_{k} s_{i} s_{x} \cdots s_{h} s_{m} s_{q} \cdots s_{v} s_{n} .
$$

To show that the replacements indicated in $u_{2}$ and $u_{2}^{\prime}$ are correct we note first that the first cycles in $u_{2}$ and $u_{2}{ }^{\prime}$ are the same, since $s_{2}$ is commutative with each of its powers. Also, since $s_{h}=s_{k} s_{2}$ and $s_{i}=s_{2} s_{k}$, we have $s_{2} s_{h}=s_{i} s_{2}$. Therefore $s_{i}$ is replaced in $u_{2}$ by what $s_{h}$ is in $u_{2}{ }^{\prime}$. Moreover, if $s_{h}$ is replaced by $s_{v}$ in $u_{2}, s_{i}$ by $s_{x}$ in $u_{2}{ }^{\prime}, s_{x}$ by $s_{q}$ in $u_{2}$ and $s_{v}$ by $s_{n}$ in $u_{2}{ }^{\prime}$, it results that $s_{m}$ is replaced by $s_{n}$ in $u_{2}$ and by $s_{q}$ in $u_{2}{ }^{\prime}$. In fact, the product $u_{2} u_{2}^{\prime}$ gives $s_{h}$ replaced by $s_{n}$, while in $u_{2}^{\prime} u_{2}$ we have $s_{h}$ replaced by the product $s_{m} s_{2}$. Hence $s_{m} s_{2}=s_{n}$. Similarly, we have that $s_{m}$ is replaced by $s_{q}$ in $u_{2}{ }^{\prime}$. From this we see that the substitution

$$
t=\left(s_{h} s_{i}\right)\left(s_{v} s_{x}\right)\left(s_{n} s_{q}\right)
$$

transforms $u_{2}$ into $u_{2}^{\prime}$ so far as the elements involved are concerned. Continuing this, we ultimately obtain a substitution $t$ of order 2 that transforms any power of $u_{2}$ into the same power of $u_{2}{ }^{\prime}$. Consider next the substitutions $u_{j}$ and $u_{j}^{\prime}$ represented by the rows $G s_{j}$ and $s_{j} G$ respectively $\left(s_{j}\right.$ not in the cyclic subgroup generated by $s_{2}$ ). In $u_{j}$ we have $s_{2}$ replaced by what $s_{j}$ is in $u_{2}^{\prime}$ and in $u_{j}^{\prime}$ we have $s_{2}$ replaced by what $s_{1}$ is in $u_{2}$. Now $G$ and $G^{\prime}$ are simply isomorphic and this isomorphism may be established such that all the substitutions begin with $s_{2}$. Then $t$ transforms $G$ into a group that is simply isomorphic with $G^{\prime}$ and such that the first two letters in corresponding substitutions are the same. Since $G$ and $G^{\prime}$ are regular it follows that $t$ transforms $G$ into $G^{\prime}$.

2. In the substitution $u_{2}$ of $G$ we have the cycle

$$
s_{k} s_{k} s_{2} s_{k} s_{2}^{2} s_{k} s_{2}^{3} \cdots s_{k} s_{2}^{e-1},
$$

where $s_{k} s_{2}{ }^{i}$ is to be considered as one letter. Let $s_{p}=s_{k} s_{2} s_{k}^{-1}$. Then, since $s_{p}{ }^{i}=s_{k} s_{2}{ }^{i} s_{k}{ }^{-1}$, we have $s_{p}{ }^{i} s_{k}=s_{k} s_{2}{ }^{i}$, that is, in the substitution of $G^{\prime}$ corresponding to the row $s_{p} G$ will be found the above cycle. This shows that the substitutions of 
$G$ and $G^{\prime}$ are composed of the same cycles combined differently when $G$ is not abelian. Take for example,

$$
\begin{aligned}
& G=1,(a b c)(d e f),(a c b)(d f e),(a d)(b f)(c e), \\
& (a e)(b d)(c f),(a f)(b e)(c d), \\
& G^{\prime}=1,(a b c)(d f e),(a c b)(d e f),(a d)(b e)(c f), \\
& (a e)(b f)(c d),(a f)(b d)(c e) .
\end{aligned}
$$

URBANA, ILLINOIS, September 24, 1918.

\section{CORRECTIONS.}

Professor G. Loria has kindly pointed out the fact that the curves discussed in the first part of my article "Some Algebraic Curves" published in volume 25, pages 85-87 of the Bulletin are special cases of curves discussed in his treatise "Spezielle Algebraische und Transcendente Ebene Kurven," volume I, pages 390-4 (1910). However the main theorem of the section, viz., the $r$ th polar of $B$ with respect to $C_{n}$ is $C_{n-r}$ is not found in Loria's treatise.

J. H. Weaver.

On page 472 of the Bulletin for July, 1918, line 10, for certain functions $t$ read certain functions of $t$; line 4 from bottom, for $l^{2 i \pi / p}$ read $e^{2 i \pi / p}$.

On page 53 of the Bulletin for November, 1918, line 11 from bottom, for field read fluid. On page 56, line 4, for $\tanh (\mu u)$ read $\tanh \left(\frac{1}{2} \mu u\right)$.

\section{NOTES.}

The total membership of the American Mathematical Society on January 1, 1919, was 723, including 79 life members. The total attendance of members at all meetings held in 1918, including sectional meetings, was 222 ; the number of papers read was 137. The number of members attending at least one meeting was 155 . Accessions to the Library in 1918 included 74 periodicals and 12 non-periodicals, making a total 